\title{
Microbiology of liver and spleen abscesses
}

\author{
I. BROOK and E. H. FRAZIER
}

Departments of Pediatrics and Infectious Disease, Naval Hospital, Bethesda, MD, USA

\begin{abstract}
To study the aerobic and anaerobic microbiology of liver and spleen abscesses and correlate the results with predisposing factors, potential causes and routes of infection, clinical and laboratory data of 48 patients with liver abscesses and 29 with spleen abscesses treated between 1970 and 1990 were reviewed retrospectively. In liver abscesses, a total of 116 isolates ( 2.4 isolates/specimen) was obtained; 43 were aerobic and facultative species (0.9 isolates/specimen) and 73 were anaerobic species or microaerophilic streptococci (1.5 isolates/specimen). Aerobic bacteria only were isolated from $12(25 \%)$ abscesses, anaerobic bacteria only from eight $(\mathbf{1 7 \%})$, and mixed aerobic and anaerobic bacteria from $28(58 \%)$; polymicrobial infection was present in $38(79 \%)$. The predominant aerobic and facultative isolates were Escherichia coli (11 isolates), Streptococcus group D (8), Klebsiella pneumoniae (5) and Staphylococcus aureus (4). The predominant anaerobes were Peptostreptococcus spp. (18 isolates), Bacteroides spp. (13), Fusobacterium spp. (10), Clostridium spp. (10) and Prevotella spp. (4). There were 12 isolates of micro-aerophilic streptococci. $S$. aureus and $\beta$-haemolytic streptococci were associated with trauma; Streptococcus group D, $K$. pneumoniae and Clostridium spp. with biliary disease; and Bacteroides spp. and Clostridium spp. with colonic disease. In splenic abscesses, a total of 56 isolates (1.9 isolates/specimen) was obtained; 23 were aerobic and facultative species $(0.8$ isolates/specimen), 31 were anaerobic species or micro-aerophilic streptococci (1.1 isolates/specimen) and two were Candida albicans. Aerobic bacteria only were isolated from nine (31\%) abscesses, anaerobic bacteria from eight $(28 \%)$, mixed aerobic and anaerobic bacteria from $10(34 \%)$ and $C$. albicans in two $(7 \%)$; polymicrobial infection was present in $16(55 \%)$. The predominant aerobic and facultative isolates were $E$. coli (5 isolates), Proteus mirabilis (3), Streptococcus group D (3), K. pneumoniae (3) and $S$. aureus (4). The predominant anaerobes were Peptostreptococcus spp. (11 isolates), Bacteroides spp. (5), Fusobacterium spp. (3) and Clostridium spp. (3). S. aureus, $K$. pneumoniae and Streptococcus group D were associated with endocarditis, $E$. coli with urinary tract and abdominal infection, Bacteroides spp. and Clostridium spp. with abdominal infection and Fusobacterium spp. with respiratory infection.
\end{abstract}

\section{Introduction}

Liver and spleen abscess are still potentially lifethreatening diseases, despite advances in their diagnosis and treatment. The most common causes of liver abscess are biliary and colonic disease, haematogenous seeding, recent gastric or duodenal surgery, local trauma and pancreatitis [1-3]. Recent studies have emphasised the polymicrobial nature of these infections with Enterobacteriaceae, Enterococcus spp. and anaerobic bacteria being the predominant isolates [1-6].

Received 13 Sept. 1997; revised version received 3 Feb. 1998; accepted 17 March 1998.

Corresponding author: Professor I. Brook, POB 70412, Chevy Chase, MD 20813, USA.
However, no correlation was made in most of these studies between the microbial isolates and the predisposing conditions [2-6]. Furthermore, the methods for isolation of anaerobic bacteria were either not specified or inadequate, which may have resulted in a failure to isolate all anaerobes present and incomplete identification of the organisms isolated [2-5]. The most common predisposing causes of splenic abscess are pyogenic infection, splenic trauma, haemoglobinopathies and contiguous disease processes extending to the spleen [7]. Staphylococci (primarily Staphylococcus aureus) and gram-negative aerobic bacteria are the most frequently reported isolates [7-11]. Polymicrobial flora are cultured from at least $10-15 \%$ of patients [711]. The reported frequency of negative culture may be up to $30 \%$ [7], which may reflect prior antibiotic 
therapy, sterile necrosis following infarction, or failure to culture properly for anaerobic and other fastidious organisms. This retrospective report describes the 20year experience in a military hospital of the diagnosis of the bacterial causes of liver and spleen abscesses.

\section{Patients and methods}

\section{Patients}

Between June 1970 and June 1990, bacteria were isolated from 48 specimens from adult patients with pyogenic liver abscess and from 29 specimens from patients with splenic abscess submitted to the clinical microbiology laboratory at the Naval Hospital in Bethesda, MD, USA.

Liver abscess. Pyogenic liver abscess was defined as one or more discrete lesions in the liver in association with positive bacterial culture of material obtained at operation or by percutaneous aspiration. Patients with amoebic or other parasitic liver abscess were excluded. Also excluded were a further nine specimens for which no clinical data were available, five specimens that showed no bacterial growth, and three specimens that were submitted in inadequate transport media for anaerobes. Clinical and microbiological data were compiled for the 48 patients of whom 23 were men; the ages of patients were in the range $23-78$ years (mean 56 years).

Splenic abscess. Splenic abscess was defined as an infectious suppurative process involving an identifiable filling defect either in the parenchyma of the spleen or in the subcapsular space. Excluded were another three splenic abscess specimens for which no clinical data were available, three that showed no bacterial growth, and two that were submitted in inadequate transport media for anaerobes. Clinical and microbiological data were compiled for the 29 patients of whom 18 were men; the ages of patients were in the range 17-81 years (mean 50 years).

Antimicrobial therapy was given to all patients with liver abscesses and all but six with splenic abscesses before sample collection. The most frequently used antimicrobial agents were amoxycillin, cefoxitin and aminoglycosides. Specimens were obtained at surgery by a swab or by percutaneous needle aspiration. The swab was placed into anaerobic transport medium (Port-Cul; BBL Microbiological Systems, Cockeysville, MD, USA) and cultures were generally inoculated within $2 \mathrm{~h}$ of collection. Needle aspiration was performed with an 18- or 20-gauge needle attached to a 5 - or $10-\mathrm{ml}$ syringe. The syringe was immediately sealed and was usually transported to the laboratory within $30 \mathrm{~min}$ of collection.

\section{Microbiological investigations}

Sheep blood agar, chocolate agar and MacConkey agar plates were inoculated for the isolation of aerobic organisms. The plates were incubated at $37^{\circ} \mathrm{C}$ aerobically (MacConkey agar) or in air with $\mathrm{CO}_{2} 5 \%$ (blood and chocolate agar) and examined after 24 and $48 \mathrm{~h}$. For the isolation of anaerobes, specimens were plated on to prereduced phytonadione-enriched Brucella blood agar, anaerobic blood agar containing kanamycin sulphate and vancomycin hydrochloride, and anaerobic blood agar containing colistin and nalidixic acid, and then the swab was placed in enriched thioglycollate broth. The agar media were incubated in anaerobic jars (BBL Microbiological Systems) and examined after 48, 96 and $120 \mathrm{~h}$. No antimicrobial susceptibility testing of anaerobic isolates was done. The thioglycollate broth was incubated for 14 days and was subcultured on to all the above media if no growth was obtained in the original cultures. Aerobic and anaerobic bacteria were identified by techniques described previously [12-14].

Blood for culture was drawn from 36 patients with liver abscess and 24 with splenic abscess, most often from an antecubital vein, after preparation of the area with povidone-iodine, and inoculated at the bedside into two bottles, one supportive of growth of aerobic bacteria and the other supportive of anaerobic bacteria.

\section{Results}

\section{Liver abscess}

Microbiology. A total of 116 isolates (2.4/specimen) was obtained from the 48 abscesses; 43 were aerobic and facultative species ( 0.9 isolates/specimen) and 73 (1.5/specimen) were anaerobes or micro-aerophilic streptococci (Table 1). Aerobic bacteria only were isolated from $12(25 \%)$ of the abscesses, anaerobic bacteria only from eight $(17 \%)$ and mixed aerobic and anaerobic bacteria from $28(58 \%)$. Polymicrobial infection was present in $38(79 \%)$ abscesses and, in these, the number of isolates varied from two to five.

The predominant aerobic and facultative isolates were Escherichia coli (11 isolates), Streptococcus group D (8), Klebsiella pneumoniae (5), $\alpha$-haemolytic streptococci (5), $S$. aureus (4) and $\beta$-haemolytic streptococci (three; two of group A and one of group F). The predominant anaerobes were: Pepiostreptococcus spp. (18 isolates) that included $P$. micros (7), P. prevotii (4), $P$. anaerobius (3), $P$. magnus (2) and $P$. asaccharolyticus (2); Bacteroides spp. (13) that included B. fragilis (7), B. thetaiotaomicron (3), B. vulgatus (2) and B. distasonis (1); Fusobacterium spp. (10) that included $F$. nucleatum (5), F. mortiferum (1) and $F$. necrophorum (1); Clostridium spp. (10) that included $C$. perfringens (5), $C$. ramosum (2) and $C$. septicum (2); Prevotella spp. (4) that included Pr. intermedia (2), Pr. melaninogenica (1) and Pr. disiens (1). There were 12 isolates of micro-aerophilic streptococci - Str. intermedius (3), Str. constellatus (3) and Gemella morbillorum (2). 


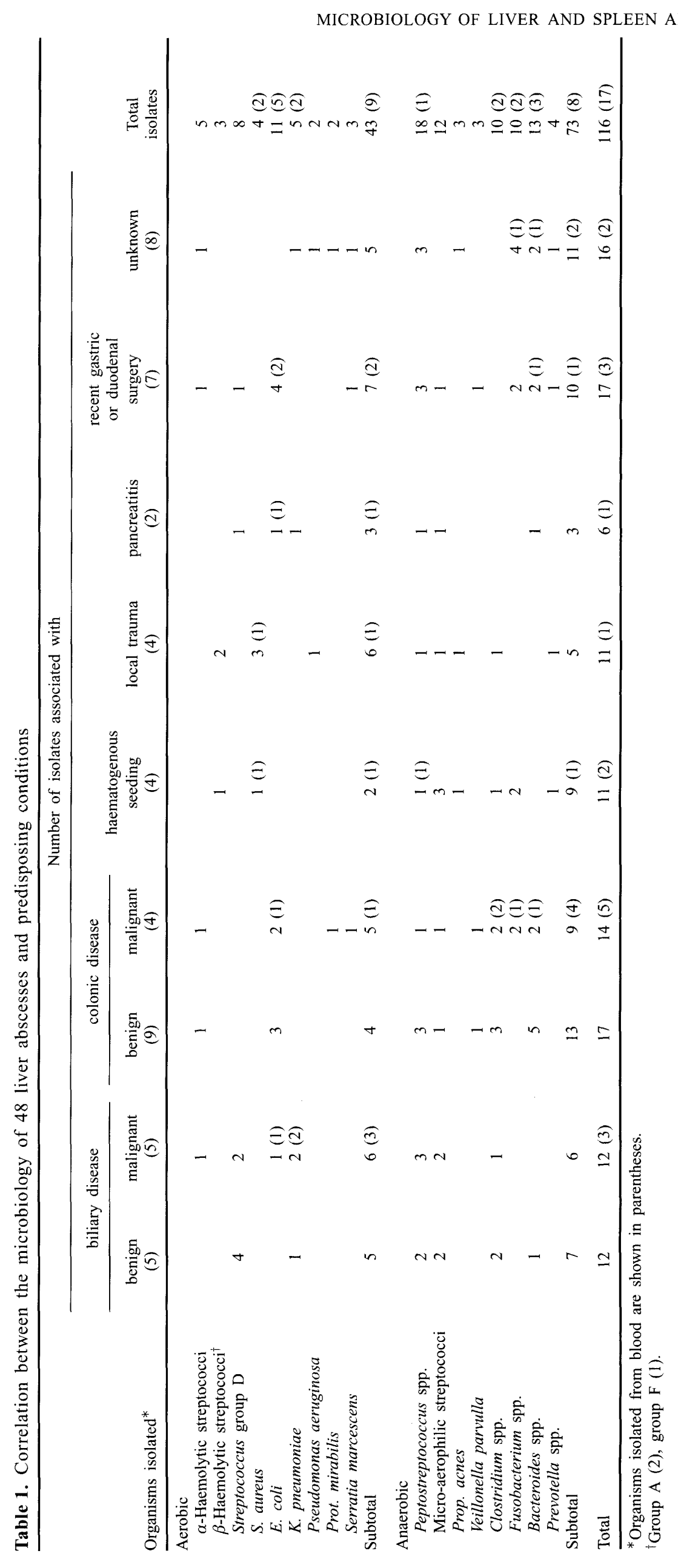


Seventeen isolates similar to those isolated from the abscesses were also cultured from $14(39 \%)$ of the 36 cases in which blood cultures were collected (Table 1).

Some relationships between the organisms isolated and the potential causes and routes of infection were noted (Table 1). S. aureus and $\beta$-haemolytic streptococci were mostly associated with trauma, Streptococcus group $\mathrm{D}$ and $K$. pneumoniae with biliary disease, Bacteroides spp. with colonic disease, and Clostridium spp. with colonic and biliary diseases. There was no correlation between the microbiological findings and the presence of a solitary ( 37 cases) or multiple abscesses (11 cases) or the location of the solitary abscesses.

Management and outcome. Antimicrobial therapy was given to all patients and surgical drainage was performed in $41(85 \%)$. The antimicrobial agents used were an aminoglycoside (43 cases), clindamycin (20), metronidazole (15), amoxycillin (12), cefoxitin (5), methicillin (5), vancomycin (4), ceftazidime (3), imipenem-cilastatin (3), ticarcillin-clavulanate (2) and cefazolin (1). Three of the patients died from their infection; one had a pancreatic carcinoma, one a colonic carcinoma and E. coli sepsis, and one had gangrenous cholecystitis and colon perforation.

\section{Spleen abscesses}

Microbiology. A total of 56 isolates (1.9/specimen) was obtained from 29 abscesses; 23 were aerobic and facultative species (0.8 isolates/specimen), 31 (1.1/ specimen) were anaerobes (or micro-aerophilic streptococci) and two were Candida albicans (Table 2). Aerobic bacteria only were isolated from nine $(31 \%)$ of the abscesses, anaerobic bacteria only from eight $(28 \%)$, mixed aerobic and anaerobic bacteria from 10 $(34 \%)$ and C. albicans from two (7\%). Polymicrobial infection was present in $16(55 \%)$ abscesses and in these the number of isolates varied from two to five.

The predominant aerobic and facultative bacteria were E. coli (5 isolates), S. aureus (4), and Streptococcus group D, K. pneumoniae and Proteus mirabilis (3 each). The predominant anaerobic bacteria were: Peptostreptococcus spp. (11 isolates) that included $P$. magnus (3), P. micros (2), $P$. prevotii (2), $P$. anaerobius (1) and $P$. asaccharolyticus (1); Bacteroides spp. (5) that included B. fragilis (2), B. vulgatus (2) and B. thetaiotaomicron (1); Clostridium spp. (3) that included $C$. perfringens (2) and C. ramosum (1); Prevotella spp. (3) that included Pr. intermedia (2) and Pr. melaninogenica (1); F. nucleatum (3); and Propionibacterium acnes (3).

Seventeen isolates similar to those isolated from the abscesses were also cultured from $13(54 \%)$ of the 24 patients from whom blood cultures were collected (Table 1).

Some relationships between the isolates and the predisposing condition were noted (Table 2). $S$. aureus, K. pneumoniae and Streptococcus group D were mostly associated with endocarditis, $E$. coli with urinary tract and abdominal infections, Bacteroides spp. and Clostridium spp. with abdominal infection, Fusobacterium spp. with respiratory tract infection and $C$. albicans with cancer and chemotherapy.

Management and outcome. All patients received antimicrobial therapy in association with splenectomy. The antimicrobial agents used were aminoglycosides (19 cases), methicillin (8), clindamycin (8), ceftazidime (6), amoxycillin (3), cefoxitin (3), imipenem-cilastatin (3), metronidazole (2), vancomycin (2), ticarcillin-clavulannate (2), ciprofloxacin (2), ceftriaxone (2), cefotaxime (2) and amphotericin-B (2). Three patients died from their infection; one patient had monocytic leukaemia and $C$. albicans abscesses and sepsis, one a generalised peritonitis and myocardial infarction, and one had endocarditis due to $S$. aureus.

\section{Discussion}

This study demonstrates the isolation of aerobic and anaerobic bacteria from liver and spleen abscesses. Organisms similar to those isolated in this study from liver abscesses have been reported by other investigators [2-6]. However, in contrast to previous reports, anaerobic bacteria were isolated more frequently from liver abscesses and their isolation was associated with colonic and biliary origins.

This study also confirms previous reports of splenic abscesses [7-11] where $S$. aureus, $K$. pneumoniae and Streptococcus group D were isolated from abscesses associated with endocarditis and $E$. coli was isolated in association with urinary tract or intra-abdominal infections. This study also emphasises the importance of anaerobic bacteria in polymicrobial splenic abscesses. As was demonstrated in previous studies [7-11], the isolation of these organisms correlates with predisposing factors that allow the dissemination of anaerobic bacteria from another infected site to the spleen. These infections can be either chronic respiratory infections caused by anaerobic bacteria, such as retropharyngeal abscess, or abdominal infection.

The high isolation rate of anaerobic bacteria in this series may be the result of the particular efforts made to use proper methods of collection and transportation of specimens and the inclusion in the report only of specimens specifically submitted for culture for both aerobic and anaerobic bacteria. Many of the previous studies did not use such methods. 


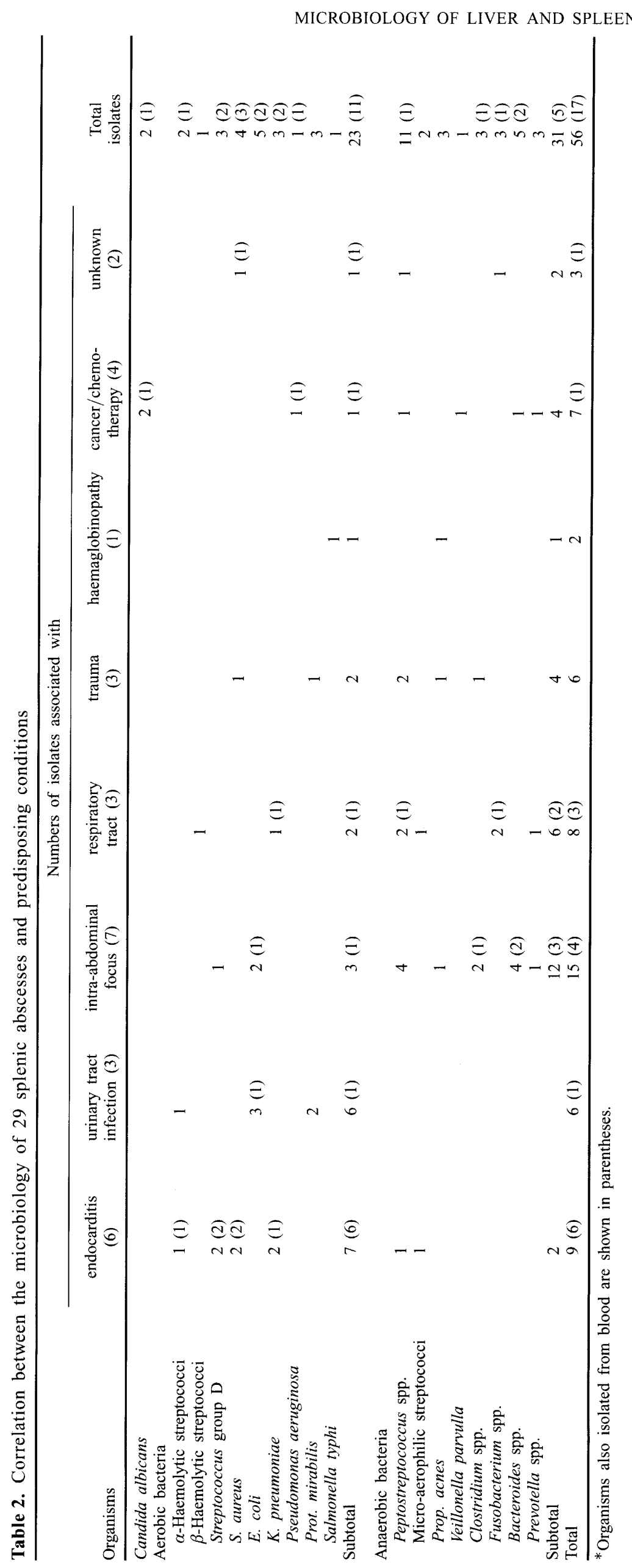


Because anaerobes are the predominant organisms present in the normal flora of the oral cavity and the gastrointestinal tract, outnumbering aerobes at a ratio of at least 100 to 1 in the oropharynx [15] and 1000 to 1 in the colon [16], their predominance in abscesses that originate from these flora is not surprising. The liver and spleen may be invaded by pyogenic microorganisms in several ways. Embolic abscesses, usually multiple, may originate from foci anywhere in the body via the arterial route. Direct extension of infection, or extension by way of the lymphatics, may develop from infected intra-abdominal sites such as appendicitis, diverticulitis, perforated bowel or pelvic infection. Extrahepatic biliary obstruction and cholangitis may cause liver abscess. Occasionally, an abscess may follow penetrating or blunt trauma.

Pyogenic liver abscesses may require percutaneous aspiration to aid in the microbiological diagnosis and to guide antibiotic therapy [17]. As a result of the development of better imaging techniques for guidance, open surgical drainage is now rarely necessary. However, care must be taken to avoid puncture of vital intra-abdominal structures and percutaneous aspiration should not be done when contra-indicated, e.g., in bleeding disorders, amoebic abscesses, or small multiple abscesses. Antimicrobial therapy should be based on the microbiological information, but if the patient's condition is unstable or fails to respond to antimicrobial therapy, surgical drainage should be performed. Evacuation of the abscess activity serves two major purposes: to obtain good bacteriological specimens to enable maximal antibiotic efficiency; and to remove and prevent local spread of purulent material. Appropriate broad-spectrum antibiotics should also be started parenterally and continued for at least 4-6 weeks. A careful attempt should be made to identify the causative micro-organisms, including anaerobes, and as many of the isolated anaerobic bacteria produce $\beta$-lactamase and are resistant to penicillins [18], antimicrobial agents effective against these organisms should be used. If anaerobic organisms are isolated, metronidazole, clindamycin, imipenem, cefoxitin or the combination of a penicillin and a $\beta$-lactamase inhibitor are the drugs of choice [1920]. Metronidazole is also a very potent amoebicide. An aminoglycoside, a quinolone, or a third-generation cephalosporin should be added if gram-negative enteric bacteria are present, and if $S$. aureus is present, anti-staphylococcal agents should be used. Antimicrobial agents, especially when used without surgical drainage, should be given for at least 6-8 weeks. A shorter course, of 4-6 weeks, may be used when good surgical drainage has been achieved, but more precise recommendations for the treatment of liver and spleen abscesses have not yet been determined in prospective studies.

We thank the staff of the clinical microbiology laboratories and the wards at the Navy Hospital in Bethesda and the secretarial assistance of Joanie Pietrafitta and Helen Wojciechowski.

The opinions and assertions contained herein are the private ones of the writers and are not to be construed as official or reflecting the views of the US Navy Department or the US Naval Service at large.

\section{References}

1. Rustgi AK, Richter JM. Pyogenic and amebic liver abscess. Med Clin North Am 1989; 73: 847-858.

2. Gyorffy EJ, Fry CF, Silva J, McGiahan J. Pyogenic liver abscess. Diagnostic and therapeutic strategies. Ann Surg 1987; 206: $699 \ldots 705$

3. Branum GD, Tyson GS, Branum MA, Meyers WC. Hepatic abscess. Changes in etiology, diagnosis, and management. Ann Surg 1990; 212: 655-662.

4. Shimada H, Ohta S, Maehara $M$, Katayama $K$, Note $M$, Nakagawara G. Diagnostic and therapeutic strategies of pyogenic liver abscess. Int Surg 1993; 78: 40-45.

5. McDonald MI, Corey GR, Gallis HA, Durack DT. Single and multiple pyogenic liver abscesses: natural history, diagnosis and treatment, with emphasis on percutaneous drainage. Medicine 1984; 63: $291-302$.

6. Sabbaj J, Sutter VL, Finegold SM. Anaerobic pyogenic liver abscess. Ann Intern Med 1972; 77: 629-638.

7. Chun CH, Raff MJ, Contreras RV et al. Splenic abscess. Medicine 1980; 59: 50-65.

8. Chulay JD, Lankerani MR. Splenic abscesses. Report of 10 cases and review of the literature. $A m J$ Med 1976; 61: $513-521$.

9. Westh H, Reines E, Skibsted L. Splenic abscesses: a review of 20 cases. Scand J Infect Dis 1990; 22: 569-573

10. Alan JO. Splenic abscess: pathogenesis, clinical features, diagnosis, and treatment. Curr Clin Top Infect Dis 1994; 14: $23-51$.

11. Nelken N, Ignatius J, Skinner M, Christensen N. Changing clinical spectrum of splenic abscess. A multicenter study and review of the literature. Am J Surg 1987; 154: 27-34.

12. Holdeman LV, Cato EP, Moore WEC et al. Anaerobe laboratory manual, 4th edn. Blacksburg, VA, Virginia Polytechnic Institute and State University. 1997.

13. Sutter VL, Citron DM, Finegold SM. Wadsworth bacteriology manual, 4th edn. Belmont, CA, Star Publishing Co. 1985

14. Lennette EH, Balows A, Hausler WJ, Shadomy $\mathrm{CH}$. Manual of clinical microbiology, 4th edn. Washington, DC, American Society for Microbiology. 1985.

15. Gibbons RJ. Aspects of the pathogenicity and ecology of the indigenous oral flora of man. In: Balows A, De Haan RM, Dowell VR, Guze LB (eds) Anaerobic bacteria: role in disease. Springfield, IL, Charles C Thomas. 1974: 267-285.

16. Gorbach SL. Intestinal microflora. Gastroenterology 1971; 60: $1110-1129$.

17. Bertel CK, van Heerden JA, Sheedy PF. Treatment of pyogenic hepatic abscess: surgical vs. percutaneous drainage. Arch Surg 1986; 121: 554-558

18. Sutter VL, Finegold SM. Susceptibility of anaerobic bacteria to 23 antimicrobial agents. Antimicrob Agents Chemother 1976; 10: $736-752$.

19. Brook I. Pediatric anaerobic infection: diagnosis and management, 2nd edn. St Louis, MO, CV Mosby. 1989.

20. Finegold SM. Anaerobic bacteria in tiuman disease. New York, Academic Press. 1977. 